\title{
INVERSE RESPONSE BEHAVIOUR IN THE BRIGHT RING RADIUS MEASUREMENT OF THE Czochralski PROCESS I: INVESTIGATION
}

\author{
Halima Zahra Bukhari ${ }^{1}$, Morten Hovd ${ }^{1}$, and Jan Winkler ${ }^{*}$ \\ ${ }^{1}$ Department of Engineering Cybernetics, NTNU, Trondheim, Norway \\ ${ }^{2}$ Institute of Control Theory, Faculty of Electrical \& Computer Engineering, TU Dresden, Germany
}

\begin{abstract}
This is the first part of a two-article series that deals with the investigation of the anomalous behaviour in the radius measurement signal of the Czochralski $(\mathrm{Cz})$ process and its mitigation in a feedback control system. The inverse or anomalous behaviour is indeed a measurement signal response, which initially is opposite to that of the expected response. This is a crucial and limiting factor in feedback control system design. The paper presents the development of a rigorous 3D raytracing method to investigate the inverse response behaviour in the measurement signal. The results of this study provide an insight into the dynamic behaviour of the $\mathrm{Cz}$ growth process. It can serve as a guideline for achieving effective crystal radius control, which is addressed in the second part of this article series.
\end{abstract}

\section{INTRODUCTION}

The Czochralski $(\mathrm{Cz})$ crystal growth process is a wellestablished and highly automated method, that is indeed a workhorse for the commercial production of monocrystalline silicon ( $\mathrm{Si}$ ) ingots. It also plays an important role in the growth of germanium and oxide crystals. The crystallization of the mono-crystalline ingots takes place inside a $\mathrm{Cz}$ puller assembly as sketched in Fig. 1. In case of Si crystal growth the feed material is first melted inside a rotatable quartz crucible. For that purpose heaters surround the crucible from all sides, including the base. Once Si is heated up to a temperature slightly higher than its melting point, the growth of a crystal ingot is then initiated by immersing a seed crystal into the melt and then gradually pulling it upwards. The pulling rod that supports the seed crystal is not just pulled upwards but also rotated - usually in the direction opposite to that of crucible rotation. Also the crucible itself is lifted gradually such that the solid-liquid interface of the growing crystal is kept in a fixed position. The pulling of a crystal from the melt results in a slightly raised liquid volume that extends from the growing crystal interface to the flat melt surface. This volume is denoted as the meniscus throughout this paper. Fig. 2 shows a schematic view. The dynamics of the meniscus is most important for the growth of the crystal.

The growth of a crystal ingot starts with a thin neck. After achieving the desired neck size, the neck stage gradually transitions into the body stage by going through intermediate stages of crown and shoulder growth. Out of different ingot segments, it is the cylindrical segment (crystal body), that is processed later by the device manufacturers.

The primary performance objective of the $\mathrm{Cz}$ process is to attain a uniform cross-section throughout the body length. Variations in crystal cross-section are commonly referred to as pinches. Crystal structure defects, such as the number of inclusions, nonuniform dopant distributions, etc. have a much higher propensity to occur at the pinch locations [27]. Therefore, to avoid pinch formation, a uniform crystal growth rate and crosssection is desired. This requires precise tracking of the crystal pulling speed and the heater temperatures such that the meniscus is influenced in a targeted manner. Especially in the body

\footnotetext{
*Corresponding author: jan.winkler@tu-dresden.de
}

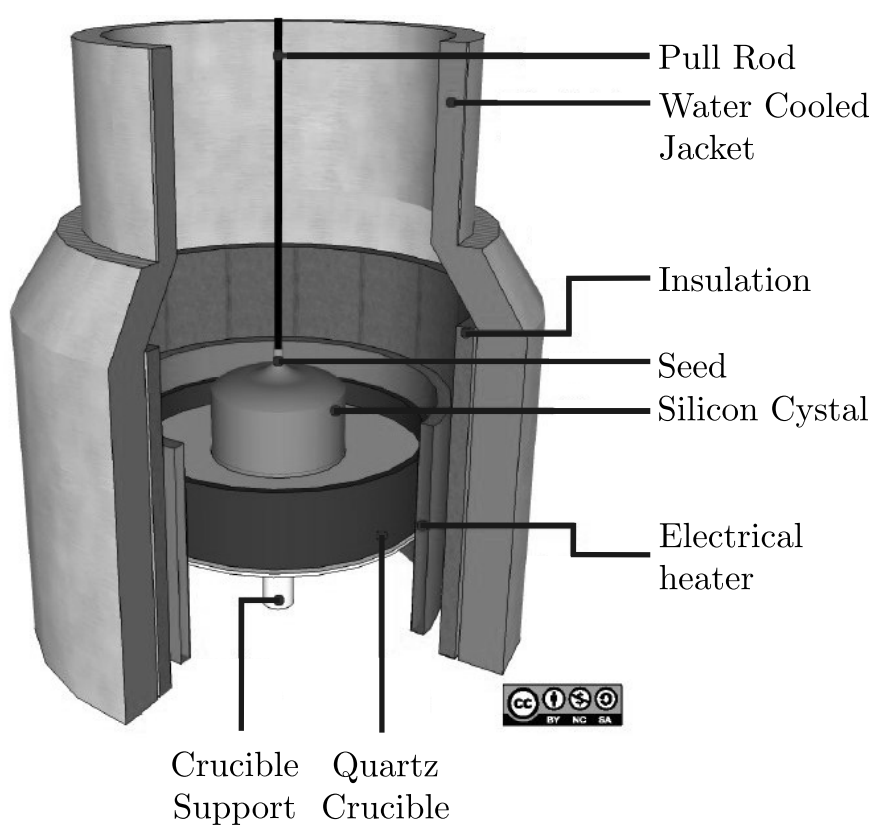

Figure 1: Assembly of a typical $\mathrm{Cz}$ puller [24].

phase the crystal diameter should remain constant. Unfortunately, the process is highly sensitive with respect to any disturbances acting on it. Accordingly, it is a challenging task to design a reliable and robust automated growth control system.

\subsection{Measurement anomaly}

Any automated feedback control system needs measured quantities in order to gain information about the current state of the process, especially the deviation of the quantities to be controlled from their set point values (i.e., the crystal radius and the growth rate). In the $\mathrm{Cz}$ process, the measured variable generally used for the feedback control of the crystal radius can either be the force acting on a load cell connected with the upper end of the pulling rod (usually referred to as weight mea- 


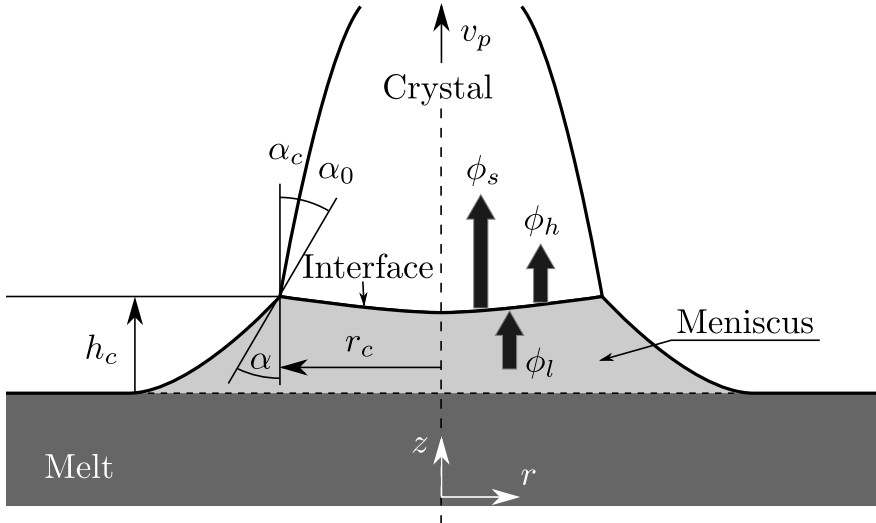

Figure 2: Schematic view of the melt-crystal interface.

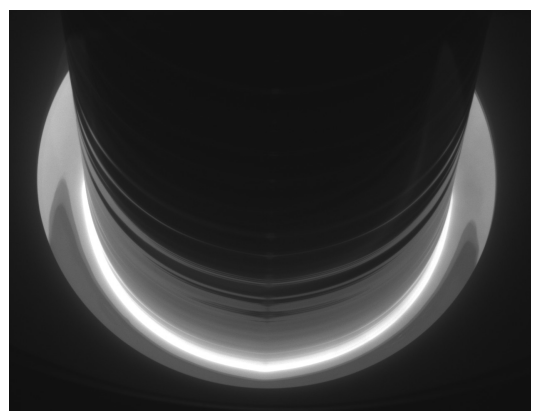

Figure 3: Actual image of the plant, captured by a CCD camera while focusing on the melt surface within the $\mathrm{Cz}$ growth furnace. The complete view of the bright circular ring on the meniscus is occluded by the crystal ingot in the centre. However, the radius of this bright ring serves as an estimate for the crystal radius.

surement $\left.^{2}\right)[20,1,2]$ or the radius measurement of a CCD camera mounted at the top of the plant and looking downward into the vessel (Fig. 3) [23, 6, 21, 8]. Since the boundary between crystal and the melt is indistinguishable, the CCD-camera is adjusted to continuously focus the meniscus in the vicinity of the three-phase boundary. It optically senses the radius of a specific bright ring formed on the meniscus. The bright annular rings on the meniscus are caused by the reflection of light by the curved meniscus in such a way that the hotter crucible wall and the heat shield underside form varying brightness pattern on the illuminated meniscus. Any clearly and consistently identifiable point on this brightness pattern, illuminated by a specific component within the hot zone, may serve as a basis for the crystal radius measurement. Therefore, in the jargon of crystal growers, this very radius measurement is termed as the bright ring radius denoted by $r_{b r}$. Fig. 3 shows an image of the illuminated meniscus as captured with a CCD camera. It is apparent that the bright ring image is a view-occluded glowing ring as some of the meniscus reflections from the opposite side of the camera are obscured either by the heat shield or the cylindrical ingot in the center or both.

${ }^{2}$ In fact it measures the weight of the crystal and the forces resulting from the surface tension and hydrostatic pressure of the meniscus.
The idea behind the weight measurement method is that the change of crystal mass per time unit divided by the pulling speed is proportional to the square of the crystal radius. For that purpose, the measured weight signal is differentiated with respect to time and used as an indirect value for the controlled variable. However, since the measured weight signal is also affected by the meniscus dynamics this equality does not hold, especially during changes of the radius. For example, in the case of a crystal radius increase the vertical component of the meniscus' surface tension decreases. Additionally, the melt column below the crystal (responsible for the static pressure) decreases and - because the density of the melt is larger than that of the solid - another decreasing effect is added. As a consequence, the measured variable initially responds inversely to the radius. This anomaly in the measured signal is depicted in Fig. 4 (middle) for a change of the crystal radius in a positive direction. This fact is well known and widely investigated in the Cz crystal growth literature $[3,14,11,9]$.

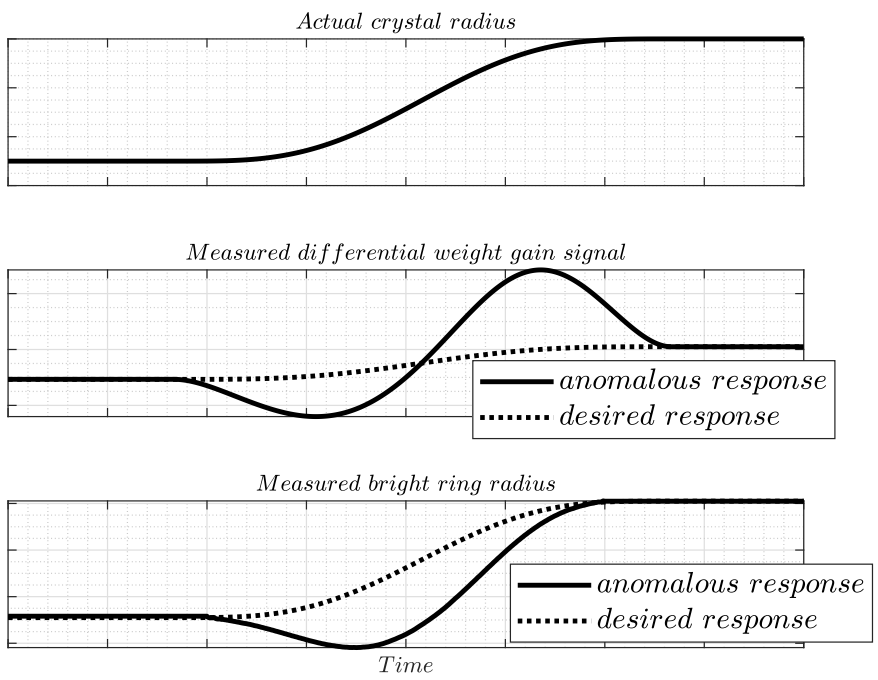

Figure 4: Illustration of anomalous behaviour in the bright ring and weight measurement signals.

A similar anomalous behaviour is known to be possessed by the bright ring measurement in a qualitative sense [9]. For example, with a decrease in pulling speed, the meniscus height decreases, making the meniscus profile flatter. This effect will result in an increase in the crystal radius, while at the same time causing the camera to initially detect a decrease in the bright ring radius. Only after the crystal radius has grown significantly will the camera detect an increase in the bright ring radius. Fig. 4 (bottom) pictorially illustrates this anomalous behaviour measuring the bright ring radius. Although there are publications dealing with the calculation of the bright ring diameter [18] a thorough investigation of the impact of the anomalous behaviour on control system performance remains. This work, in particular, aims at investigating the aforementioned from both systematic and quantitative standpoint.

For this purpose, the first part of this two-article series contains a detailed investigation of the phenomena causing the anomalous behaviour. The second part of the series describes the control design to mitigate the detrimental effects of the anomalous measurement behaviour on the control of crystal radius. Some of the results of this two-article series have previously been pre- 
sented in an abridged form for to a control audience in conference publications [4,5]. This two-article series aims to present the results in full detail and accessible also to a crystal growth audience, without requiring expert knowledge of control.

\subsection{Paper organization}

Section 2 gives an overview of the control of the Czochralski process as it is currently done in an industrial environment. A simplified process model describing the overall $\mathrm{Cz}$ dynamics appropriate for control design is derived in Section 3 comprising a rigorous description of the crystal growth dynamics (section 3.1) and simplified temperature dynamics (section 3.2). A method based on ray-tracing for the bright ring radius estimation and inverse response investigation is presented in section 4 . Finally, Section 5 provides conclusions and points to further work to be presented in part II of this article series.

\section{Conventional control of the Cz Process using OPTICAL DIAMETER MEASUREMENT}

The challenging task in control system design is that the $\mathrm{Cz}$ process is a complex process with a combination of both faster as well as slower dynamics. The dynamics at the crystallization interface controlled by the pulling speed is quite fast. It can affect the crystal radius, meniscus height and the corresponding growth conditions quickly compared to the slower dynamics associated with the heater temperature input and the highly complex nonlinear heat transfer phenomena. The heat transfer from the heaters to the crystallization front undergoes both lag and long time delays. Thus, the effect of heat input on the crystal growth rate is noticeable after a significant time lapse.

In conventional $\mathrm{Cz}$ control these different dynamics are addressed by a cascaded controller structure comprising three control loops: The automatic diameter controller (ADC), the automatic growth rate controller (AGC) and the automatic temperature controller (ATC), respectively [19], (cf. Fig. 5).

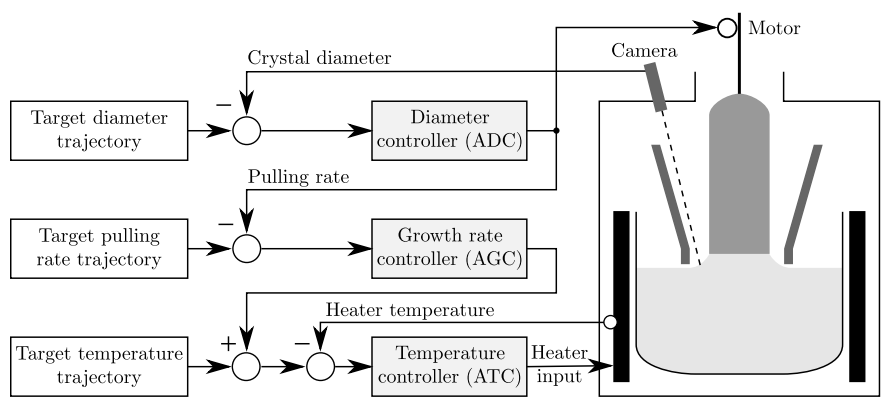

Figure 5: Schematics showing a conventional control structure of the $\mathrm{Cz}$ system.

During a typical growth cycle in the body stage, a target temperature trajectory is applied to the temperature controller. In an actual process, the target temperature trajectory has an increasing trend to compensate for the following events occurring throughout the growth cycle within the $\mathrm{Cz}$ growth chamber: (i) A gradual uplift of the crucible, therefore progressively reducing the crucible exposure to the heaters. (ii) With the ongoing crystallization, the crystal continues to protrude into the colder areas above the heat shield, thereby increasing the heat transfer away from the interface. The temperature controller takes its measurement from a pyrometer. There is a small opening in the containment structure that allows for pyrometer insertion, providing the pyrometer with a view of graphite lining surrounding the heater element. This thereby provides a measurement for the heater temperature.

The growth rate controller and the temperature controller are connected in series with each other. The tracking error for the pulling speed triggers the growth rate controller, which in turn, adds a trim value to the target temperature trajectory. Due to sluggish dynamics from heater power to crystal growth rate, a time-varying target temperature trajectory is introduced. The intent of introducing the target temperature trajectory is to counteract the factors described above that change the heat transfer characteristics, and thereby, provide anticipative action to reduce variations in the crystal growth rate. A well-designed temperature trajectory implies better tracking of pulling speed and will therefore reduce the contribution from the growth rate controller.

\section{A LOW ORDER MODEL OF THE CZ DYNAMICS}

For a rigorous investigation of the bright ring anomaly and its impact on the control system performance, a basic model of the $\mathrm{Cz}$ dynamics is required. The Czochralski process comprises of crystal growth dynamics at the interface explained in detail in Section 3.1 and additionally of the heater/ temperature dynamics which significantly affects the outcome of the process. Especially the growth rate $v_{g}$ driving the basic growth dynamics is a result of the thermal situation at the crystallization interface. However, an accurate model representing the temperature dynamics and the complex heat transfers within the $\mathrm{Cz}$ process would have to be derived from describing the system using partial differential equations, and thereafter applying discretization on a fine grid to arrive at a large set of ordinary differential equations representing the temperature dynamics $[7,12]$. While such a model may be appropriate for process design studies, it is commonly considered too large and impractical for control design studies. In Section 3.2, we will therefore develop two simplified models instead, based on a coarse lumped model, adapted from the model presented in [24]. While the heat transport is of little importance for the investigation of the bright ring measurement in Section 4.5 of this paper, it is central when evaluating control performance in a qualitatively reasonable manner in part II of this article series. Therefore, Section 3.2 can be skipped by readers interested only in the analysis of the bright ring measurement.

\subsection{Growth dynamics}

The standard $\mathrm{Cz}$ growth model commonly referred to as either the hydromechanical-geometrical model [29] or simply the crystal growth dynamics at the crystal-melt solidification inter- 
face, is given by (cf. Fig. 2 also):

$$
\begin{aligned}
\dot{r}_{c} & =v_{g} \tan \left(\alpha_{c}\right) \\
\dot{h}_{c} & =v_{p}-v_{g} \\
r_{b r} & =f_{b r}\left(r_{c}, h_{c}\right) \\
\alpha_{c} & =\arcsin \left\{1-\left(\frac{h_{c}}{a}\right)^{2}\left[1+0.6915\left(\frac{r_{c}}{a}\right)^{-1.1}\right]\right\}-\alpha_{0} \\
v_{g} & =\frac{\phi_{s}-\phi_{l}}{\rho_{s} \Delta H}
\end{aligned}
$$

where $r_{c}$ is the crystal radius, $h_{c}$ is the height of the meniscus at the three-phase boundary, $v_{p}$ is the pulling speed, $v_{g}$ is the growth rate of the crystal (in axial direction assuming a flat solid-liquid interface) and $\alpha_{c}$ is the cone angle at the interface. Finally, the output $r_{b r}$ is the bright ring radius obtained from the camera image. Its dependence on the crystal radius and the meniscus height can be expressed in general by the function $f_{b r}$. That this approach is reasonable will be shown later in this paper. With reference to (1e), $\phi_{s}$ is the heat flux from the interface into the crystal, while $\phi_{l}$ is the heat flux from the meniscus to the interface. $\rho_{s}$ is the density of the solid crystal, and $\Delta H$ is the specific latent heat of fusion. The derivative of the meniscus height expressed in (1b) assumes perfect compensation for melt level changes through crucible lift.

The expression of $\alpha_{c}$ in (1d) is derived from the analytical approximation of meniscus height $h_{c}$ given by [17]. The overall growth angle is expressed as $\alpha=\alpha_{0}+\alpha_{c}$, where $\alpha_{0}$ is the contact angle at constant radius growth, i.e., $\alpha_{c}=0$. We will assume $\alpha_{0}=11^{\circ}[26,25]$. The Laplace constant $a$, also termed the capillary length, is given by $a=\sqrt{\frac{2 \sigma_{L G}}{\rho_{L} \cdot g}}$ with the specific surface tension $\sigma_{L G}$ of the Si melt, the Si melt mass density $\rho_{L}$ and gravitational acceleration $g$. One has a value of $a=7.62 \mathrm{~mm}$ as calculated for Si with $\sigma_{L G}=0.732 \mathrm{~N} \mathrm{~m}^{-1}$ and $\rho_{L}=2570 \mathrm{~kg} \mathrm{~m}^{-3}$

Under the steady-state growth conditions, the heat flux $\phi_{l}$ entering the interface from the meniscus, the heat flux $\phi_{h}=\varrho_{s} \Delta H v_{g}$ released due to phase change and the heat flux $\phi_{s}$ directed into the crystal are balanced. The crystal growth rate (1e) is defined on the basis of this heat balance. The heat balance across the crystallization interface should be maintained such that the net flow of heat is towards the crystallizing interface. In other words, the continuous heat loss from the meniscus into the crystal (heat of fusion/latent heat) ensures the ongoing crystallization/solidification [29].

\subsection{Heater/Temperature dynamics}

The total path for the heat transfer, from the heaters to the interface, is divided into control volumes. A control volume is a fictitious volume with constant physical properties. For each control volume a heat balance can be established from which an ordinary differential equation for its temperature can be deduced. In the following, two different assumptions are used as a basis for describing the mode of heat transfer and resulting in an expression for $\phi_{l}$ in (1e).

I. The heat transfer from the bulk of the melt into the meniscus is caused by convection, while the heat transfer across the meniscus itself is based on pure conduction. This results in a model consisting of four control volumes (cf. Fig. 6a). In this model, the heat flux entering the solid-liquid interface from the melt will depend on the meniscus height, as will be shown in Section 3.2.1.

II. The heat transfer from the bulk of the melt to the interface is based on convection only resulting in a model consisting of three control volumes (cf. Fig. 6b). Here, the varying meniscus height does not have any influence on the heat flux entering the solid-liquid interface (cf. section 3.2.2).

Though these two assumptions are in a sense two extremes, however, the actual heat transfer across the meniscus is likely to be a combination of both convection and conduction. Thus, a control design that works for both models can be expected to work for the heat transfer mode(s) occurring in the actual process.

As in our previous work $[4,5]$, we will refer to the resulting models as Model I and Model II, respectively.

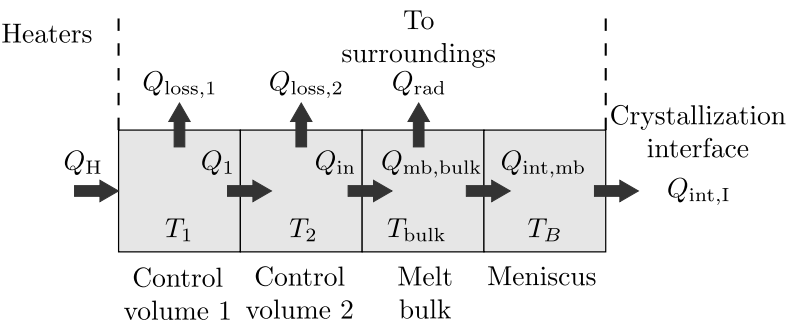

(a) Model I: Conduction based heat transport across the meniscus.

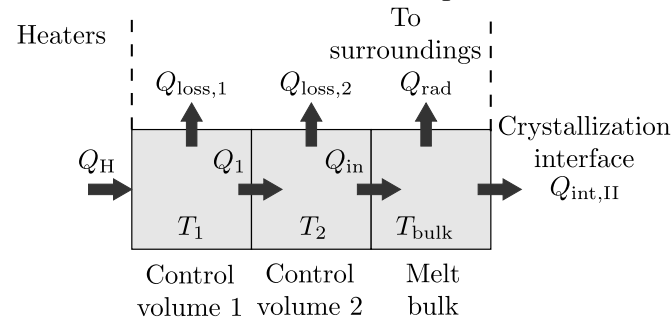

(b) Model II: Convection based heat transport across the meniscus.

Figure 6: Illustration of the lumped heat transport models with their control volumes.

\subsubsection{Heat transport in Model I}

In the case of Model I (cf. Fig. 6a), the first two control volumes are used to provide a coarse second order approximation to the dynamics of the heat transfer from the heater to the melt. The temperature $T_{1}$ of control volume 1 may roughly correspond to the temperature measured by the pyrometer on the graphite lining surrounding the heaters, while the second control volume of fictional temperature $T_{2}$ introduces an additional time lag representing the thermal inertia of the inner assemblies surrounding the crucible. Their dynamics is given by

$$
\begin{aligned}
& \dot{T}_{1}=\frac{Q_{H}-Q_{1}-Q_{\text {loss }, 1}}{\tau_{1}} \\
& \dot{T}_{2}=\frac{Q_{1}-Q_{\text {in }}-Q_{\text {loss }, 2}}{\tau_{2}}
\end{aligned}
$$


where $\tau_{1}, \tau_{2}$, represent the parameters proportional to timedelay for the heat transfers through control volumes 1 and 2, respectively [25]. These parameters are chosen to approximate an effective time delay of $600 \mathrm{~s}$ for each of the two control volumes. In (2) one has the heat input $Q_{H}$ from the heaters, i.e., the manipulated variable for the ATC controller, the thermal energy $Q_{1}$ that enters the control volume 2 from control volume 1 and the thermal energy $Q_{i n}$ that enters the melt. These heat transfer rates are modelled using [25]

$$
Q_{1}=\beta_{1}\left(T_{1}-T_{2}\right)
$$

and

$$
Q_{\text {in }}=\beta_{2}\left(T_{2}-T_{\text {bulk }}\right) \text {, }
$$

with the overall heat transfer coefficients $\beta_{1}, \beta_{2}$ between the control volumes and the melt bulk with temperature $T_{b u l k}$. The two heat losses $Q_{l o s s, 1}$ and $Q_{l o s s, 2}$ in (2) are assumed to be constant for a shorter time scale of dynamical analysis.

The last two control volumes to the right of this model (cf. Fig. 6a) distinctively represent the melt bulk region and the meniscus, respectively. The heat transfer is modelled as two heat transfers in series: the convective heat transfer from the bulk of the melt into the meniscus and the conductive heat transfer across the meniscus to the crystallization interface. The dynamics of the melt bulk temperature $T_{\text {bulk }}$ reads

$$
\dot{T}_{\text {bulk }}=\frac{Q_{\text {in }}-Q_{\text {int }, I}-Q_{\text {rad }}}{V_{s} \cdot \rho_{l} \cdot C_{p}},
$$

where $V_{s}, \rho_{l}$ and $C_{p}$ define the melt volume, the density and the specific heat capacity of liquid $\mathrm{Si}$, respectively. The convection based heat flow $Q_{m b, b u l k}$ from the melt bulk region into the meniscus of temperature $T_{B}$ can be calculated from

$$
Q_{m b, b u l k}=\beta_{\text {conv }, I}\left(T_{\text {bulk }}-T_{B}\right),
$$

with the overall heat transfer coefficient $\beta_{c o n v, I}$.

The conductive heat flow $Q_{i n t, m b}$ from the meniscus to the crystallization interface (with Si melting point temperature $T_{S}$ ) reads

$$
Q_{i n t, m b}=\frac{k_{c o n d, I} A_{i}\left(T_{B}-T_{S}\right)}{h_{c}},
$$

where $A_{i}=\pi r_{c}^{2}$ is the cross-sectional area of the solidification interface and $k_{c o n d, I}$ is the heat conductivity of liquid $\mathrm{Si}^{3}$. Due to the short height of the meniscus it is reasonable - on the timescale of relevance for crystal growth - to neglect the dynamics of $T_{B}$, i.e., $\dot{T}_{B}=0$, and assume the two heat transfers $Q_{i n t, m b}$ and $Q_{m b, b u l k}$ to be equal. This allows us to eliminate $T_{B}$ from (6),(7) and arrive at the following expression for the overall heat flow $Q_{i n t, I}$ entering the crystallization interface from the bulk:

$$
Q_{\text {int }, I}=\beta_{\text {int }}\left(T_{\text {bulk }}-T_{S}\right)
$$

\footnotetext{
${ }^{3}$ As can be seen in Eq. (7), the temperature gradient is assumed to be $\left(T_{B}-T_{S}\right) / h_{c}$ which is a quite rough but common approximation in lumped parameter models of the $\mathrm{Cz}$ process [16]. In reality, the thickness of the thermal boundary layer is the driving force for conductive heat transfer. But since this layer is not modelled here this approximation is used. It simply reflects the heuristic assumption that the closer the interface to the hot melt, the more the crystallization is inhibited [22].
}

The coefficient $\beta_{\text {int }}=\left(\beta_{c o n v, I}^{-1}+h_{c} k_{c o n d, I}^{-1} A_{i}^{-1}\right)^{-1}$ is the overall heat transfer coefficient that combines the two coefficients from expressions (6) and (7) in series. Similarly (from (6),(7)) the heat transfer coefficient $\beta_{c o n v, I}$ is given by

$$
\beta_{\text {conv }, I}=\frac{k_{\text {cond }, I}\left(T_{B, 0}-T_{S}\right) A_{i}}{h_{c}\left(T_{b u l k, 0}-T_{B, 0}\right)},
$$

using initial steady-state values $T_{B, 0}, T_{b u l k, 0}$ for $T_{B}, T_{b u l k}$, respectively. Hence, its value is assumed constant throughout the simulations. Moreover, $\beta_{c o n v, I}$ is adjusted to achieve the observed crystal growth rate.

Finally, the radiative heat loss $Q_{\text {rad }}$ from the melt surface is expressed as:

$$
Q_{\text {rad }}=A_{f m} F_{m c} \epsilon_{m} \sigma\left(T_{\text {bulk }}^{4}-T_{\text {env }}^{4}\right)
$$

where $A_{f m}$ is the free melt surface area expressed as $A_{f m}=$ $\pi\left(R_{c r u}^{2}-r_{c}^{2}\right), F_{m c}$ is the radiation view factor considering the heat radiation from the free melt surface to the crystal surroundings, $\epsilon_{m}$ is the melt emissivity, $\sigma$ is the Stefan-Boltzmann constant and $T_{e n v}$ is the temperature of the environment.

\subsubsection{Heat transport in Model II}

In case of model II convective heat transport from the melt to the crystallization interface is considered only, i.e., the bulk of the melt, as well as the temperature of the meniscus, are at the same temperature level. Hence, the model consists of only three control volumes (cf. Fig. 6b).

The temperature dynamics of the first two control volumes is given by (2), while the bulk melt temperature $T_{\text {bulk }}$ is given by:

$$
\dot{T}_{\text {bulk }}=\frac{Q_{\text {in }}-Q_{\text {int }, I I}-Q_{\text {rad }}}{V_{s} \cdot \rho_{l} \cdot C_{p}} .
$$

In the above equation (11), the radiative heat loss $Q_{\text {rad }}$ is formulated as in Eq. (10), while $Q_{\text {int,II }}$, the convective heat transfer from the melt bulk to the crystallization interface, is given as

$$
Q_{\text {int }, I I}=\beta_{\text {conv }, I I}\left(T_{\text {bulk }}-T_{S}\right),
$$

where $\beta_{\text {conv }, I I}$ represents the convective heat transfer from the bulk of the melt to the crystallization interface. The value of $\beta_{\text {conv }, I I}$ given by

$$
\beta_{c o n v, I I}=\frac{\phi_{l} A_{i}}{\left(T_{b u l k, 0}-T_{S}\right)}
$$

is adjusted to achieve the observed crystal growth rate.

\subsection{Overall model}

With the results from sections 3.1 and 3.2 the overall $\mathrm{Cz}$ dynamics, including both growth and temperature dynamics, can be written in the so-called state-space form with the state $\mathbf{x}=$ $\left(r_{c}, h_{c}, T_{\text {bulk }}, T_{1}, T_{2}\right)^{T}$ :

$$
\begin{aligned}
\dot{\mathbf{x}} & =\left(\begin{array}{c}
v_{g} \tan \left(\alpha_{c}\right) \\
v_{p}-v_{g} \\
\left(Q_{\text {in }}-Q_{\text {int }}-Q_{\text {rad }}\right) /\left(V_{s} \rho_{l} C_{p}\right) \\
\left(Q_{H}-Q_{1}-Q_{\text {loss }, 1}\right) / \tau \\
\left(Q_{1}-Q_{\text {in }}-Q_{\text {loss }, 2}\right) / \tau
\end{array}\right)=\mathbf{f}(\mathbf{x}, \mathbf{u}) \\
\mathbf{y} & =\left(\begin{array}{c}
r_{b r} \\
T_{1}
\end{array}\right), \quad \mathbf{u}=\left(\begin{array}{c}
v_{p} \\
Q_{H}
\end{array}\right) .
\end{aligned}
$$


In (13a), $Q_{\text {int }}$ can either be $Q_{\text {int,I }}$ or $Q_{\text {int,II }}$ depending on the choice of the heater model. The growth rate $v_{g}$ is calculated according to (1e) with $\phi_{l}=Q_{\text {int }} / A_{i}$. In this model, $u$ indicates the input vector comprising of two manipulating inputs $\left(v_{p}, Q_{H}\right)$, while the measured output $y$ comprises of the bright ring radius $r_{b r}$ and the temperature $T_{1}$ sensed by the pyrometer. Note, that in case of model I the growth rate depends on the meniscus height (cf. (7), (8)). A method for determining $r_{b r}$, proposed in the next section, is the main topic of this paper.

\section{RAY-TRACING METHOD FOR BRIGHT RING RADIUS ESTIMATION}

In this section a rigorous ray-tracing simulation, combined with the crystal growth dynamics, is developed to simulate the camera image of the illuminated meniscus. The simulated camera image is then used to calculate the bright ring radius for control system design and analysis. In [18], ray-tracing is used to estimate the bias between the actual crystal radius and the measured bright ring radius for a static case. This paper presents a method to simulate the actual camera image and the dynamic analysis of the resultant bright ring radius measurement that can aid in effective and improved control system design.

The light incident on the meniscus from different components in the hot zone assembly gets reflected from the meniscus surface and captured by the camera as a bright ring image. Fig. 7 shows a simplified ray-tracing setup. It presents the vertical cross-sectional view of the $\mathrm{Cz}$ growth furnace (crucible wall, heat-shield, and camera location). For the sake of simplicity, only one ray from each source is shown to be incident onto the meniscus and reflected thereof before reaching the camera. Though the rays may undergo multiple reflections before reaching the camera, an instance of the ray reflected twice from the meniscus is shown by a dashed line in the same figure.

It is clear that a key factor when modeling the dynamics of the bright ring radius is the knowledge of the meniscus shape. The meniscus shape can be calculated from the so-called LaplaceYoung equation that accounts for surface tension, gravity, and hydrostatic pressure to express the shape and height of the meniscus. Unfortunately, there is no analytical solution to the Laplace-Young equation, which, therefore, has to be solved numerically [13]. An alternative to the aforementioned approach is the use of an analytical approximation of the meniscus shape.

\subsection{Meniscus shape approximation}

An approximation of the meniscus profile based on the actual crystal radius $r_{c}$ at the interface and the meniscus height $h_{c}$ is presented in [15]:

$$
\begin{aligned}
r\left(h_{c}, r_{c}, z\right) & =r_{c}+\sqrt{\frac{2}{A}-h_{c}^{2}}-\sqrt{\frac{2}{A}-z^{2}} \\
& -\frac{1}{\sqrt{2 A}} \ln \left[\frac{z}{h_{c}} \cdot \frac{\sqrt{2}+\sqrt{2-A \cdot h_{c}^{2}}}{\sqrt{2}+\sqrt{2-A \cdot z^{2}}}\right]
\end{aligned}
$$

where $r$ and $z$ are the radial and vertical coordinates of the meniscus surface, respectively. Thus, one has $(r, z)=\left(r_{c}, h_{c}\right)$ where the meniscus connects to the crystal, while roughly $(r, z)=\left(R_{c r u}, 0\right)$ at the crucible wall. The parameter $A$ is defined as

$$
A=\frac{1}{a^{2}}+\frac{\cos (\alpha)}{2 r_{c} h_{c}} .
$$

This meniscus profile can be extended to define a full 3D meniscus surface by rotating the profile about the $z$ axis, i.e., $360^{\circ}$ along the azimuthal plane.

The ray-tracing simulation relies primarily on the knowledge of the $\mathrm{Cz}$ growth model parameters $\left(r_{c}, h_{c}\right)$ as discussed in Section 3.1 combined with the information about the meniscus shape profile (14) to simulate the image of the CCD camera.

\subsection{Hot zone geometry}

The aspects of the hot zone geometry used in describing the ray-tracing method are shown in Figs. 7 and 8. The reference

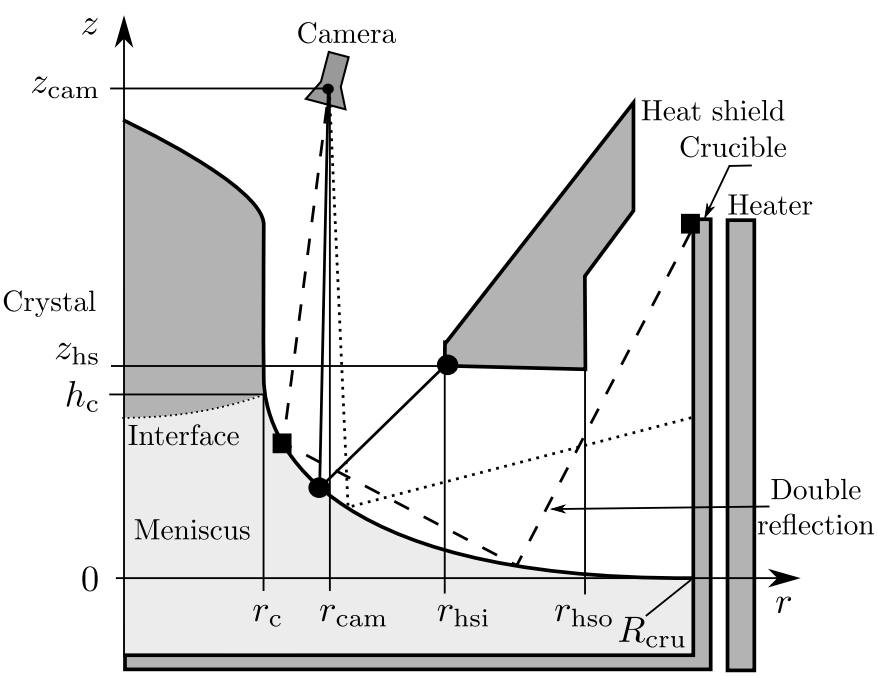

Figure 7: Ray-tracing set up showing incident and reflected light rays within the growth furnace.

frame origin $O$ is placed at the level of the free melt surface such that the horizontal $x$-axis and the lateral $y$-axis form the $x y$-plane spanning the base of the meniscus, while the $z$-axis extends vertically along the center of the ingot. The camera is located in the $x z$-plane $(y=0)$. In terms of cylindrical coordinates, the camera location is in the $r z$-plane, i.e., in the $0^{\circ}$ azimuthal plane. The hot zone components, such as the crucible wall and annular heat shield surrounding the growing crystal, have dimensions defined as:

- Crucible radius: $R_{c r u}$

- The coordinates for the heat shield underside are described in terms of its height as well as inner and outer radii given by:

- $z_{h s}$ is the height of the heat shield underside w.r.t. the free melt surface

- $r_{h s i}$ is the inner radius of the heat shield underside w.r.t. O

- $r_{h s o}$ is the outer radius of the heat shield underside w.r.t. O 
- The camera location w.r.t. $O$ is described by the position vector $\overrightarrow{\mathbf{p}}_{c}$ such that: $\overrightarrow{\mathbf{p}}_{c}=x_{c a m} \hat{i}+0 \hat{j}+z_{c a m} \hat{k}^{4}$, where

- $z_{\text {cam }}$ is the height of the camera w.r.t. the free melt surface

- $x_{\text {cam }}$ is the radial location of the camera, i.e., $x_{\text {cam }}=r_{\text {cam }}$ as $y_{\text {cam }}=0$

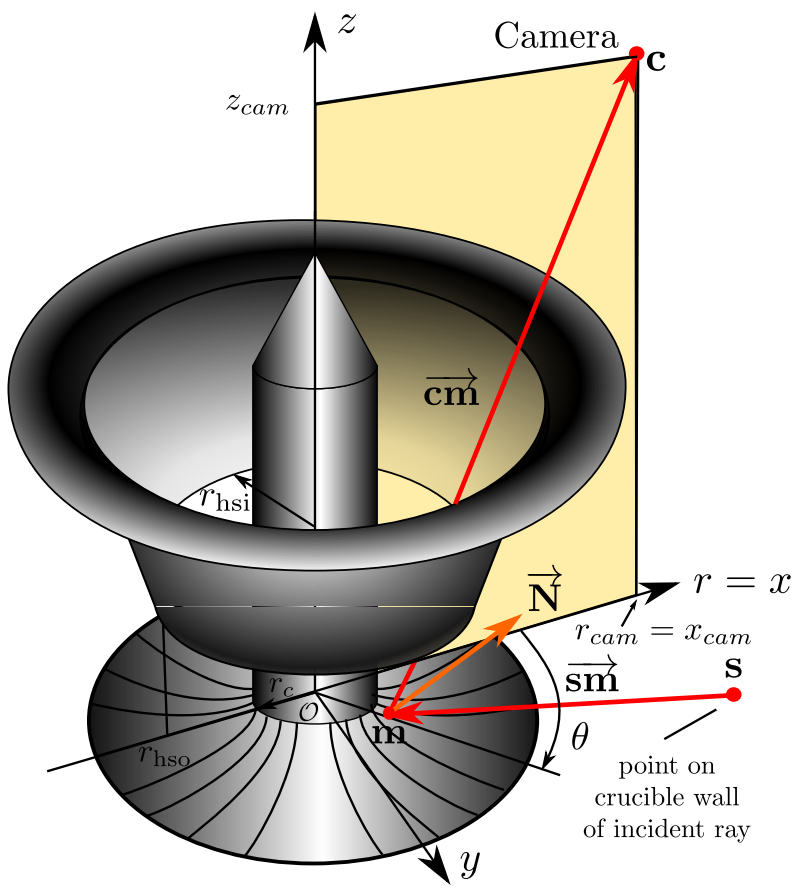

Figure 8: 3D ray-tracing scheme featuring an instance of an incident ray $\mathbf{s} \overrightarrow{\mathbf{m}}$ emerging from the crucible wall. The reflected ray from a point $(\mathbf{m})$ reaches the camera with location marked as (c). The incident and reflected rays may or may not exist in the same plane.

\subsection{Computation of tangents and normals to the meniscus surface}

Followed by the generation of 3D meniscus surface is the calculation of tangents and normals to the entire meniscus surface. This, in turn, helps to determine the incoming and outgoing rays for the camera image simulation. The tangents and the unit normals to the meniscus surface are mathematically notated by $\overrightarrow{\mathbf{T}}$ and $\overrightarrow{\mathbf{N}}$ in $\mathbb{R}^{3}$, respectively. Since the meniscus surface is axisymmetric, tangents and normals can be calculated using the 2D meniscus curve, and then rotate these around the $z$-axis to the required azimuthal orientation.

For any arbitrary point $\left(x_{m_{0}}, 0, z_{m_{0}}\right)$ (or equivalently $\left(r_{m_{0}}, 0, z_{m_{0}}\right)$ in cylindrical coordinates) on the meniscus profile such that

$4 \hat{i}, \hat{j}$ and $\hat{k}$ are the unit vectors directed along $x, y$ and $z$-axes, respectively $z_{m_{0}}=f\left(r_{m_{0}}\right)$, the tangent vector can be calculated as:

$$
\begin{aligned}
\left.\overrightarrow{\mathbf{T}}\right|_{\left(r_{m_{0}}, 0, z_{m_{0}}\right)} & =\left.\frac{1}{\sqrt{1+f^{\prime 2}(r)}}\left[\begin{array}{c}
\frac{\Delta r}{\Delta r} \\
\frac{\Delta \theta}{\Delta r} \\
\frac{\Delta f(r)}{\Delta r}
\end{array}\right]\right|_{\left(r_{m_{0}}, 0, f\left(r_{m_{0}}\right)\right.} \\
& =\frac{1}{\sqrt{1+f^{\prime 2}\left(r_{m_{0}}\right)}}\left[\begin{array}{c}
1 \\
0 \\
f^{\prime}\left(r_{m_{0}}\right)
\end{array}\right] .
\end{aligned}
$$

The normal vector is orthogonal to the tangent, and hence we have

$$
\left.\overrightarrow{\mathbf{N}}\right|_{\left(r_{m_{0}}, 0, z_{m_{0}}\right)}=\frac{1}{\sqrt{1+f^{\prime 2}\left(r_{m 0}\right)}}\left[\begin{array}{c}
-f^{\prime}\left(r_{m_{0}}\right) \\
0 \\
1
\end{array}\right] .
$$

Finally, the unit normal to any arbitrary point in question (say $\left.\mathbf{m}_{(r, \theta, z)}\right)$ can be determined by rotating $\left.\overrightarrow{\mathbf{N}}\right|_{\left(r_{m_{0}}, 0, z_{m_{0}}\right)}$ through an angle $\theta$ about the $z$-axis, such that

$$
\overrightarrow{\mathbf{N}}_{m_{\left(r_{m}, \theta, z m\right)}}=\left.R_{z}(\theta) \cdot \overrightarrow{\mathbf{N}}\right|_{\left(r_{m_{0}}, 0, z_{m_{0}}\right)},
$$

where $R_{z}(\theta)$, the rotation matrix yielding the desired rotation through an arbitrary angle $\theta$, about the $z$-axis is given as

$$
R_{z}(\theta)=\left[\begin{array}{ccc}
\cos (\theta) & -\sin (\theta) & 0 \\
\sin (\theta) & -\cos (\theta) & 0 \\
0 & 0 & 1
\end{array}\right] .
$$

\subsection{Computing incoming and outgoing rays}

A rigorous ray-tracing method that abides the laws of reflection is employed to simulate the bright ring formation over the curved meniscus. Fig. 8 illustrates the schematic of the 3D raytracing. The outgoing ray that enters the camera after reflection from a point $\mathbf{m}$ on the meniscus (described by position vector $\overrightarrow{\mathbf{p}}_{m}$ in cylindrical coordinates as $\left(r_{m}, \theta, z_{m}\right)$ or as $\left(x_{m}, y_{m}, z_{m}\right)$ in Cartesian coordinates) is given by

$$
\overrightarrow{\mathbf{c m}}=\overrightarrow{\mathbf{p}}_{c}-\overrightarrow{\mathbf{p}}_{m}={ }_{\left(x_{c a m}-x_{m}\right) \hat{i}-y_{m} \hat{j}+\left(z_{c a m}-z_{m}\right) \hat{k} .}
$$

Based on the knowledge of meniscus normals given in (17), the incoming and outgoing rays to the meniscus and camera, respectively can be determined such that the angle of incidence equals the angle of reflection, though the two angles need not to be be co-planar. For a specific point on the meniscus, the projection of the reflected ray $\overrightarrow{\mathbf{c m}}$ in the direction of normal vector $\overrightarrow{\mathbf{N}}$ at the same point is given by

$$
\operatorname{proj}_{\overrightarrow{\mathbf{N}}} \overrightarrow{\mathbf{c m}}=(\overrightarrow{\mathbf{c m}} \cdot \overrightarrow{\mathbf{N}}) \overrightarrow{\mathbf{N}},
$$

where $(\cdot)$ indicates the vector dot product operation. Similarly, the projection of $\overrightarrow{\mathbf{c m}}$ in the direction orthogonal to $\overrightarrow{\mathbf{N}}$ is given as

$$
\operatorname{proj}_{\perp \rightarrow \overrightarrow{\mathbf{N}}} \overrightarrow{\mathbf{c m}}=\overrightarrow{\mathbf{c m}}-\operatorname{proj}_{\overrightarrow{\mathbf{N}}} \overrightarrow{\mathbf{c m}}=\overrightarrow{\mathbf{c m}}-(\overrightarrow{\mathbf{c m}} \cdot \overrightarrow{\mathbf{N}}) \overrightarrow{\mathbf{N}}
$$

The orthogonal projection of incoming ray vector $\overrightarrow{\mathbf{s m}}$ is the same as the reflected ray vector $\overrightarrow{\mathbf{c m}}$ but with the opposite sign, i.e., $\operatorname{proj}_{\overrightarrow{\mathbf{N}}} \overrightarrow{\mathbf{s m}}=-\overrightarrow{\mathbf{c m}}+(\overrightarrow{\mathbf{c m}} \cdot \overrightarrow{\mathbf{N}}) \overrightarrow{\mathbf{N}}$ However, the projection of incoming ray vector in the direction of normal vector $\overrightarrow{\mathbf{N}}$ is the same as that of reflected ray vector, i.e., $\operatorname{proj}_{\overrightarrow{\mathbf{N}}} \overrightarrow{\mathbf{s m}}=(\overrightarrow{\mathbf{c m}} \cdot \overrightarrow{\mathbf{N}}) \overrightarrow{\mathbf{N}}$ 
Thus, the incoming ray from a given source point inside the hot zone, represented as $\overrightarrow{\mathbf{s m}}$, is given by

$$
\begin{aligned}
\overrightarrow{\mathbf{s m}} & =(\overrightarrow{\mathbf{c m}} \cdot \overrightarrow{\mathbf{N}}) \overrightarrow{\mathbf{N}}-\overrightarrow{\mathbf{c m}}+(\overrightarrow{\mathbf{c m}} \cdot \overrightarrow{\mathbf{N}}) \overrightarrow{\mathbf{N}} \\
& =2(\overrightarrow{\mathbf{c m}} \cdot \overrightarrow{\mathbf{N}}) \overrightarrow{\mathbf{N}}-\overrightarrow{\mathbf{c m}} \\
& =2 \overrightarrow{\mathbf{N}}\left(\overrightarrow{\mathbf{N}}^{T} \overrightarrow{\mathbf{c m}}\right)-\overrightarrow{\mathbf{c m}} \\
& =\left(2 \overrightarrow{\mathbf{N}}^{T}-\mathbf{I}\right) \overrightarrow{\mathbf{c m}},
\end{aligned}
$$

where $I$ is the identity matrix. It is noteworthy that the incident rays $\overrightarrow{\mathbf{s m}}$ and the reflected rays $\overrightarrow{\mathbf{c m}}$ may pass through planes corresponding to different azimuthal orientations. In order to determine if the source of illumination on the illuminated meniscus is the crucible wall or the heat shield underside, the following steps can be followed:

The sum of the two vectors, given by

$$
\overrightarrow{\mathbf{p}}_{s}=\overrightarrow{\mathbf{p}}_{m}+\overrightarrow{\mathbf{s m}}
$$

describes a position vector $\overrightarrow{\mathbf{p}}_{s}$ for the point of incidence, $\mathbf{s}$ w.r.t. $O$. In (23), $\overrightarrow{\mathbf{s m}}$ shall not be confused with the incoming ray (cf. Fig. 8). Instead, the expression (23) makes use of vector mathematics (head-to-tail rule of vector addition) by interpreting $\overrightarrow{\mathbf{s m}}$ as a vector that has to be extended appropriately up to the source point, as in (24).

The intersection of the incident ray with the crucible wall can be found by scaling the position vector $\overrightarrow{\mathbf{s m}}$ by a factor ' $k$ ' such that it emerges from the crucible wall of radius $R_{c r u}$. This can be achieved by solving the expression given in (24) for the positive root of ' $k$ ':

$$
\left(x_{m}+k \overrightarrow{\mathbf{s m}}_{x}\right)^{2}+\left(y_{m}+k \overrightarrow{\mathbf{s m}}_{y}\right)^{2}=R_{c r u}^{2}
$$

The elevation of the scaled up incident ray $k \overrightarrow{\mathbf{s m}}$ emerging from the crucible wall is given by its $k \overrightarrow{\mathbf{s m}}_{z}$. The double reflection (shown in Fig. 7) is caused by the incoming ray that emerges from the portion of the crucible wall lying below the melt level, i.e., the $z$-coordinate of $k \overrightarrow{\mathbf{s m}}$ is negative $(k \overrightarrow{\mathbf{s m}} z<0)$. The origin of rays undergoing double reflection can be found by calculating another reflection where the incoming ray hits the meniscus surface. The details are omitted for brevity. Note that it is possible for some rays to be reflected more than twice, especially when the meniscus close to the crystal is highly curved, which may occur when the meniscus is high. Such multiple reflections have not been considered further in this work - but the calculations required to include them in the ray tracing are more tedious than difficult.

The incident ray from the heat shield is the one whose $x$ and $y$-coordinates at $z_{h s}$ satisfy the following inequality condition:

$$
r_{h s i}<\sqrt{s m_{x}^{2}+s m_{y}^{2}}<r_{h s o}:
$$

Thus, a 3D ray-tracing simulation, based on the procedure outlined above, traces every ray that reaches the camera back to its emission point (the complete annular heat shield surrounding the growing crystal and the cylindrical crucible wall containing the molten $\mathrm{Si}$ ). The rays, which are reflected twice from the meniscus, are also included in the simulated bright ring image.
However, some of the reflections from the meniscus surface, lying on the side farther to the camera, are obscured either due to the presence of a cylindrical crystal ingot or by the heat shield. Likewise, the heat shield blocks many of the light rays which emanate from various emission points, above and beyond the heat shield underside, from reaching the camera.

The various sources of illumination on the bright ring image are depicted in Fig. 9 by different gray-scale values.

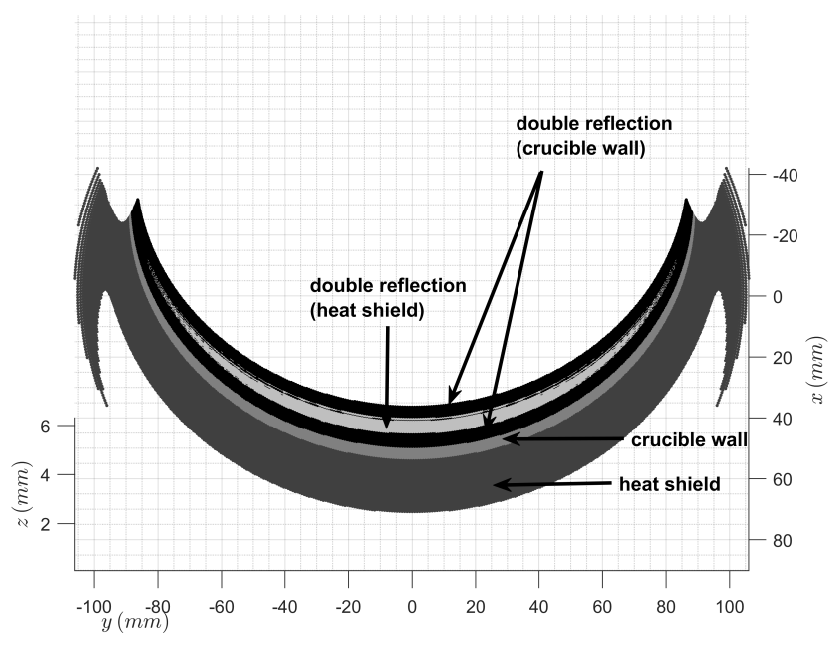

Figure 9: Meniscus image showing different regions illuminated by various components in the hot zone. The view is symmetric on either side of the $x z$-plane/camera plane ${ }^{5}$.

The brightness sensed by the camera will depend on three factors: i.) The brightness of the emitting surface. ii.) The orientation of the emitting surface relative to the direction of the emitted ray. iii.) The focusing of light caused by the curvature of the meniscus.

Accounting accurately for i.) will require knowledge both of the emissivity of the heat shield and crucible wall, as well as the temperature distribution along these surfaces. Such information is not available to the present authors, and would require the output from some very detailed simulators. Instead, it is assumed that both the underside of the heat shield and the crucible wall have uniform (and the same) brightness. Luckily, this simplification does not impede our ability to study the bright ring anomaly, as will become apparent.

Factors ii.) and iii.) are accounted for by performing small perturbations around the point on the meniscus where the ray is reflected before entering the camera. Let these perturbations define the vertices of a region on the meniscus surface, and let $A_{m}$ be the area of that region when projected in the direction of the ray $\overrightarrow{\mathbf{c m}}$. Reflection calculations are then performed to find the point of origin for each of the perturbed rays. The origins of the perturbed and reflected rays define a region on the emitting surface. Let $A_{s}$ be the area of this region of the

${ }^{5}$ The $x$ and $y$ axes in Figs. $(9 \& 10)$ define the radial coordinates of the meniscus, i.e., $r=\sqrt{x^{2}+y^{2}}$, while $z$-axis represents the height of the meniscus above the melt surface. Thus, the plane of the camera expressed in cylindrical coordinates is $(r, \theta=0, z)$ 
emitting surface, when projected in the direction of the emitting ray $\overrightarrow{\mathbf{s m}}$. A relative brightness measure ${ }^{6}$ is then found from the ratio of $A_{s}$ to $A_{m}$.

The calculated brightness profile obtained, therefore, is illustrated in Fig. 10 where the color denotes the brightness of the reflection.

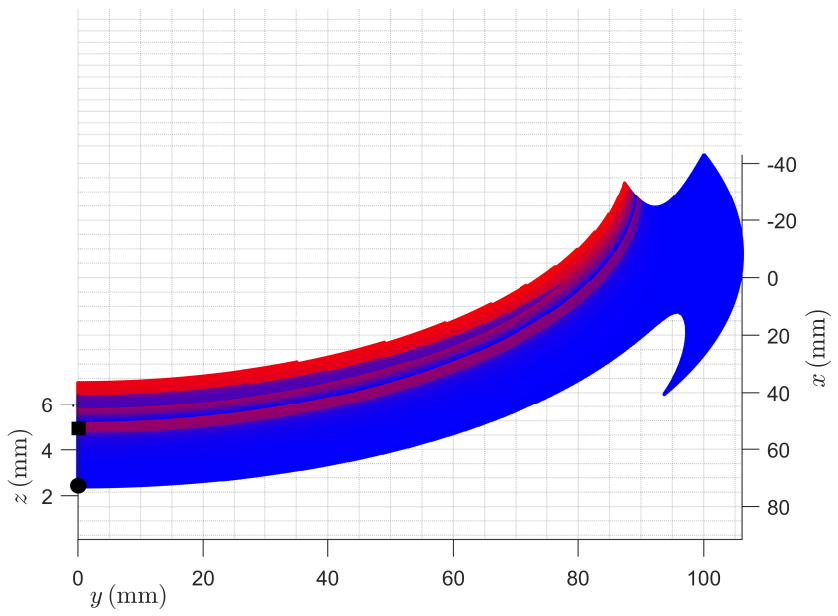

Figure 10: Theoretically calculated brightness profile as observed by the camera. Minimum brightness (blue); maximum brightness (red). Since, the view is symmetric on either side of the $x z$-plane/camera plane, the left portion of the meniscus is not shown.

In order to use a feature of the camera image for control of the crystal radius, two obvious criteria must be fulfilled: i.) the feature should be located close to the actual crystal radius, and ii.) the feature should be clearly and reliably identifiable in the camera image for all conditions that are expected during the body stage of the process (i.e., for all values of crystal radius and meniscus height that are likely to occur in the body stage). Studying the calculated reflection images (and comparing to the camera image in Fig. 3), two such features can be identified:

- The highest point on the crucible wall (same as the outermost edge of the underside of heat shield) illuminating a point ' $\square$ ' on the meniscus in Figs. $7 \& 9$.

- The innermost edge of the underside of heat shield) illuminating a point ' $\bullet$ ' on the meniscus in Figs. $7 \& 9$.

The first of these features indicated by ' $\mathbf{m}$ ' is closer to the actual crystal radius and is therefore the preferred feature to use for crystal radius control. It corresponds to the lower brightness border in the overexposed Fig. 3. Knowledge of the point from where the light that causes this feature originates, allows us to study the behaviour of the corresponding measurement under dynamical process conditions.

${ }^{6}$ Note that it would be easy to account also for the brightness of the emitting surface, if such information is available.

\subsection{Anomaly detection via $3 D$ ray-tracing simulation}

Under normal operating conditions, the physical systems rarely encounter any abrupt changes in their physical parameters/state variables. Therefore, the objective is to input a smooth crystal radius change to the ray-tracing simulation and investigate how the resultant bright ring measurement differs from the actual input signal (crystal radius). Thus, a pulling speed profile is selected ${ }^{7}$ such that it drives the $\mathrm{Cz}$ dynamics to generate an output that comprises of smooth profile for the crystal radius. Furthermore, it is worth mentioning that for this particular choice of a smooth pulling speed profile, the crystal growth rate is assumed to be constant.

Fig. 11 depicts how the chosen pulling speed profile, driving the $\mathrm{Cz}$ dynamics, results in the desired crystal radius $\left(r_{c}\right)$ variation. Besides, the same figure shows the system trajectories for meniscus height $\left(h_{c}\right)$ and cone angle $\left(\alpha_{c}\right)$.
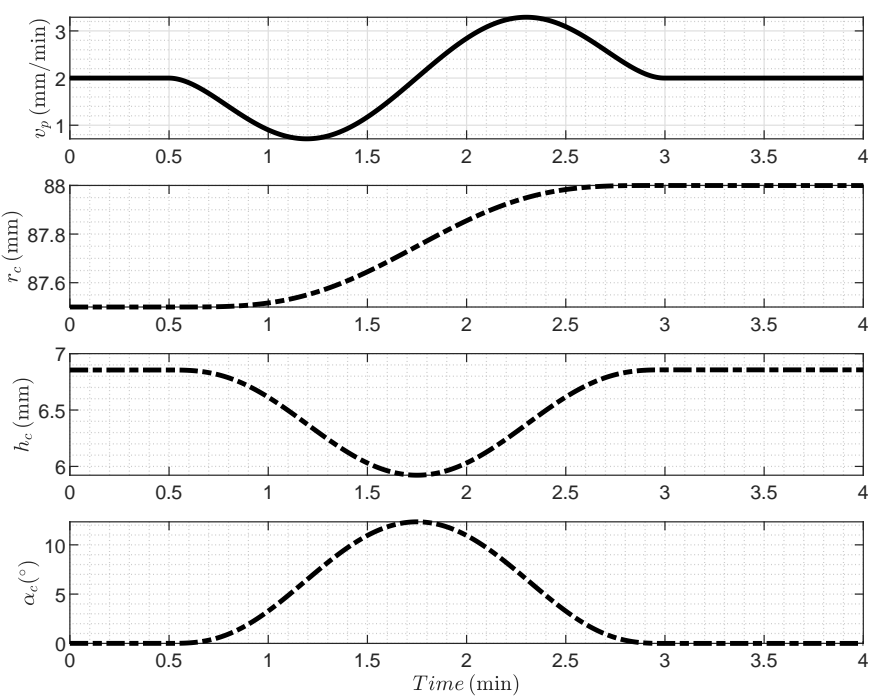

Figure 11: Smooth and continuous profile for the applied pulling speed (solid) and the resultant profiles (dash-dotted) for crystal radius, meniscus height and growth angle.

For a smoothly varying crystal radius profile (cf. second subfigure in Fig. 11), the corresponding bright ring measurement based on the ray-tracing method is carried out at various points along the highest contrast line on the 3D meniscus image. One of the aforementioned illuminated meniscus points lies in the plane of the camera $\left(0^{\circ}\right.$ azimuth), while the others lie in the planes at azimuthal orientations $10^{\circ}, 20^{\circ}, 30^{\circ}$ and $40^{\circ}$ off the camera plane.

The resultant bright ring radii responses versus the expected crystal radius $r_{c}$ response presented in Fig. 12, clearly reveal the presence of the inverse response behaviour in the measurement signal.

\footnotetext{
${ }^{7}$ Details can be seen in [28]
} 

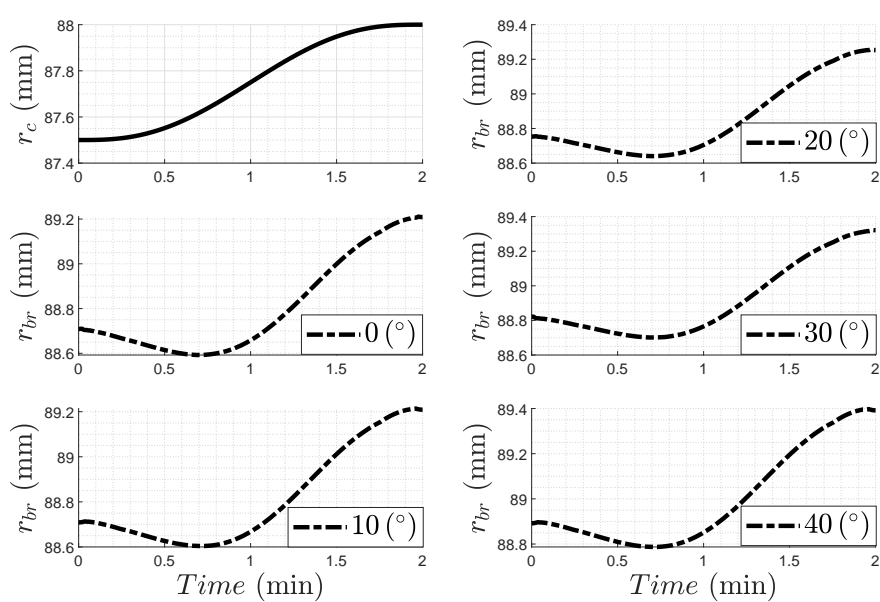

Figure 12: Actual crystal radius $r_{c}$ (solid) v.s. bright ring signal $r_{b r}$ (dotted) measured at different azimuthal orientations, indicated respectively, at the bottom right corner of each subfigure.

\section{CONCLUSIONS AND WAY FORWARD}

This work primarily focuses on a 3D ray-tracing method that simulates the glowing meniscus image captured with the CCD camera. The simulated camera image provides a reference point for the bright ring radius measurement, a crucial measure for the controlled variable. Through dynamic simulation based on the ray-tracing scheme, the exhibition of inverse response behaviour by the bright ring measurement signal is verified. This peculiar behaviour can pose fundamental limitations to the design of the $\mathrm{Cz}$ control system. The mitigation of this inverse response in the context of control, circumventing fundamental limitations with feedback control by combining feedback and parallel compensation,will extensively be dealt with in the second part of the two-article series.

\section{ACKNOWLEDGEMENTS}

This work has been funded by the Norwegian Research Council's ASICO project No. 256806/O20.

\section{REFERENCES}

[1] W Bardsley, B Cockayne, GW Green, DTJ Hurle, GC Joyce, JM Roslington, PJ Tufton, HC Webber, and M Healey. Developments in the weighing method of automatic crystal pulling. Journal of Crystal Growth, 24: 369-373, 1974.

[2] W Bardsley, DTJ Hurle, and GC Joyce. The weighing method of automatic Czochralski crystal growth: I. basic theory. Journal of Crystal Growth, 40(1):13-20, 1977.

[3] W Bardsley, DTJ Hurle, GC Joyce, and GC Wilson. The weighing method of automatic Czochralski crystal growth: II. control equipment. Journal of Crystal Growth, 40(1):21-28, 1977.

[4] Halima Zahra Bukhari, Morten Hovd, and Jan Winkler. Limitations on control performance in the Czochralski crystal growth process using bright ring measurement as a controlled variable. IFAC-PapersOnLine, 52(14):129 134, 2019. ISSN 2405-8963. 18th IFAC Symposium on Control, Optimization and Automation in Mining, Mineral and Metal Processing, MMM 2019.

[5] Halima Zahra Bukhari, Morten Hovd, and Jan Winkler. Design of Parallel Compensator and Stabilizing Controller to mitigate non-minimum phase behaviour of the Czochralski Process. IFAC-PapersOnLine, 2020. submitted to 21 st IFAC World Congress.

[6] TG Digges, RH Hopkins, and RG Seidensticker. The basis of automatic diameter control utilizing "bright ring" meniscus reflections. Journal of Crystal Growth, 29(3): 326-328, 1975.

[7] E. Dornberger, W. von Ammon, N. van den Bogaert, and F. Dupret. Transient computer simulation of a $\mathrm{Cz}$ crystal growth process. Journal of Crystal Growth, 166:452-457, 1996. doi: 10.1016/0022-0248(96)00068-1.

[8] Thierry Duffar. Crystal growth processes based on capillarity: Czochralski, Floating zone, shaping and crucible techniques. John Wiley \& Sons, 2010.

[9] Michael A Gevelber. Dynamics and control of the Czochralski process III. interface dynamics and control requirements. Journal of Crystal Growth, 139(3-4):271285, 1994.

[10] Michael A Gevelber and George Stephanopoulos. Dynamics and control of the Czochralski process: I. modelling and dynamic characterization. Journal of Crystal Growth, 84(4):647-668, 1987.

[11] Michael A Gevelber, George Stephanopoulos, and Michael J Wargo. Dynamics and control of the Czochralski process II. Objectives and control structure design. Journal of Crystal Growth, 91(1-2):199-217, 1988.

[12] Karl-Heinz Hoffmann, Axel Voigt, and Michael Metzger. Numerical simulation and control of industrial crystal growth processes. In Willi Jäger and Hans-Joachim Krebs, editors, Mathematics - Key Technology for the Future: Joint Projects between Universities and Industry, pages 331-342. Springer Berlin Heidelberg, Berlin, Heidelberg, 2003. ISBN 978-3-642-55753-8. doi: 10. 1007/978-3-642-55753-8\{ட\}27. URL https://doi. org/10.1007/978-3-642-55753-8\{_\}27.

[13] Chun Huh and LE Scriven. Shapes of axisymmetric fluid interfaces of unbounded extent. Journal of Colloid and Interface Science, 30(3):323-337, 1969.

[14] DTJ Hurle. Control of diameter in Czochralski and related crystal growth techniques. Journal of Crystal Growth, 42: 473-482, 1977.

[15] DTJ Hurle. Analytical representation of the shape of the meniscus in Czochralski growth. Journal of Crystal Growth, 63(1):13-17, 1983.

[16] DTJ Hurle, GC Joyce, M Ghassempoory, AB Crowley, and EJ Stern. The dynamics of Czochralski growth. Journal of Crystal Growth, 100(1-2):11-25, 1990.

[17] Tom H Johansen. An improved analytical expression for the meniscus height in Czochralski growth. Journal of Crystal Growth, 141(3-4):484-486, 1994. 
[18] Steven L Kimbel and Joseph A O’Sullivan. Shape estimation for online diameter calibration in Czochralski silicon crystal growth. In Process Imaging for Automatic Control, volume 4188, pages 45-57. International Society for Optics and Photonics, 2001.

[19] Kihong Lee, Dongki Lee, Jinguk Park, and Moonyong Lee. MPC based feedforward trajectory for pulling speed tracking control in the commercial Czochralski crystallization process. International Journal of Control, Automation, and Systems, 3(2):252-257, 2005.

[20] John Levinson. Temperature control for crystal pulling, October 6 1959. US Patent 2,908,004.

[21] R Lorenzini, F Nuff, and D Blair. An overview of silicon crystal growth processes. Solid State Technology, 2:33, 1974.

[22] M Neubert and J Winkler. Nonlinear model-based control of the Czochralski process IV: Feedforward control and its interpretation from the crystal grower's view. Journal of Crystal Growth, 404:210-222, 2014.

[23] EJ Patzner, RG Dessauer, and MR Poponiak. Automatic diameter control of Czochralski crystals. Semiconductor Products and Solid State Technology, 10(10):25, 1967.

[24] Parsa Rahmanpour. Model-based Control of the Czochralski Silicon Crystal Pulling Process. PhD thesis, Department of Engineering Cybernetics, NTNU, 2017.

[25] Parsa Rahmanpour, Steinar Sælid, and Morten Hovd. Run-to-run control of the Czochralski process. Computers $\mathcal{E}$ Chemical Engineering, 104:353 - 365, 2017. ISSN 0098-1354.

[26] VA Tatarchenko. Shaped Crystal Growth, volume 20. Springer Science \& Business Media, 1993.

[27] Vitali Tatartchenko. Chapter 2, The Possibility of Shape Stability in Capillary Crystal Growth and Practical Realization of Shaped Crystals. In Crystal Growth Processes Based on Capillarity: Czochralski, Floating Zone, Shaping and Crucible Techniques, pages 51 - 114. John Wiley \& Sons, Ltd, 04 2010. ISBN 978-0-470-71244-3.

[28] J Winkler, M Neubert, and J Rudolph. Nonlinear modelbased control of the Czochralski process I: Motivation, modeling and feedback controller design. Journal of Crystal Growth, 312(7):1005-1018, 2010.

[29] Jan Winkler, Michael Neubert, Joachim Rudolph, Ning Duanmu, and Michael Gevelber. Chapter 3, Czochralski Process Dynamics and Control Design. In Crystal Growth Processes Based on Capillarity: Czochralski, Floating Zone, Shaping and Crucible Techniques, pages 115-202. John Wiley \& Sons, Ltd, 2010. ISBN 9781444320237. 
Preprint - INVERSE RESPONSE BEHAVIOUR IN THE BRIGHT RING RADIUS MEASUREMENT OF THE CZOchralSki PROCESS I: INVESTIGATION

\begin{tabular}{|c|c|c|c|}
\hline Symbol & Unit & Description & Initial value \\
\hline \multicolumn{4}{|c|}{ States/ parameters common between the two thermal models } \\
\hline$Q_{H}$ & $\mathrm{~kW}$ & Heater input & 58.6 \\
\hline$Q_{1}$ & $\mathrm{~kW}$ & $\begin{array}{l}\text { Heat energy entering the control volume } 2 \text { (quartz crucible) from } \\
\text { control volume } 1 \text { (graphite crucible) }\end{array}$ & 27.3 \\
\hline$Q_{\text {in }}$ & $\mathrm{kW}$ & Heat entering the melt & 7.3 \\
\hline$Q_{\text {loss }, 1}$ & $\mathrm{~kW}$ & Heat loss from control volume 1 & 30 \\
\hline$Q_{\text {loss }, 2}$ & $\mathrm{~kW}$ & Heat loss from control volume 2 & 20 \\
\hline$Q_{\text {out }}$ & $\mathrm{kW}$ & Heat transferred away from the melt & 7.3 \\
\hline$T_{1}$ & $\mathrm{~K}$ & Intermediate temperature & 1970 \\
\hline$T_{2}$ & $\mathrm{~K}$ & Intermediate temperature & 1914 \\
\hline$T_{S}$ & $\mathrm{~K}$ & Temperature in the vicinity of crystallization interface & 1683 \\
\hline$T_{B, 0}$ & $\mathrm{~K}$ & Initial temperature of the meniscus & 1688.6 \\
\hline$T_{\text {bulk,0}}$ & $\mathrm{K}$ & Initial temperature of the melt bulk & 1704 \\
\hline$\rho_{l}$ & $\mathrm{~kg} \mathrm{~m}^{-3}$ & liquid state density of $\mathrm{Si}$ & 2570 \\
\hline$\rho_{s}$ & $\mathrm{~kg} \mathrm{~m}^{-3}$ & solid state density of $\mathrm{Si}$ & 2330 \\
\hline$C_{p}$ & $\mathrm{~J} \mathrm{~kg}^{-1} \mathrm{~K}^{-1}$ & Specific heat capacity of Si melt & 1000 \\
\hline$\Delta H$ & $\mathrm{~J} \mathrm{~kg}^{-1}$ & Latent heat of fusion & $1.79 \times 10^{6}$ \\
\hline$\phi_{s}$ & $\mathrm{~W} \mathrm{~m}^{-2}$ & Heat flux into the solid crystal from the crystallization interface & $1.3 \times 10^{5}$ \\
\hline$\phi_{l}$ & $\mathrm{~W} \mathrm{m^{-2 }}$ & Heat flux entering the meniscus & $4.6 \times 10^{4}$ \\
\hline$\epsilon_{m}$ & - & Melt emissivity [10] & 0.2 \\
\hline$\sigma$ & $\mathrm{W} \mathrm{m}^{-2} \mathrm{~K}^{-4}$ & Stefan-Boltzmann constant & $5.67 \times 10^{-8}$ \\
\hline$T_{\text {env }}$ & $\mathrm{K}$ & Temperature of the environment [10] & 1262 \\
\hline$F_{m c}$ & - & $\begin{array}{l}\text { Radiation view factor from free melt surface to the crystal sur- } \\
\text { roundings }\end{array}$ & 0.5 \\
\hline \multicolumn{4}{|c|}{ States and parameters exclusive to thermal model I } \\
\hline$T_{\text {bulk }}$ & $\mathrm{K}$ & Temperature of the melt bulk & 1704 \\
\hline$T_{B}$ & $\mathrm{~K}$ & Temperature at the base of the meniscus & 1688.6 \\
\hline$Q_{m b}$ & $\mathrm{~kW}$ & Heat entering the meniscus & 7.3 \\
\hline$k_{\text {cond,I }}$ & $\mathrm{W} \mathrm{m}^{-1} \mathrm{~K}^{-1}$ & conductivity of liquid $\mathrm{Si}$ at $1700 \mathrm{~K}$ & 57 \\
\hline \multicolumn{4}{|c|}{ States and parameters exclusive to thermal model II } \\
\hline$T_{\text {bulk }}$ & $\mathrm{K}$ & $\begin{array}{l}\text { Temperature of the melt bulk including the temperature of the } \\
\text { meniscus }\end{array}$ & 1704 \\
\hline
\end{tabular}

Table 1: Parameters/ states for thermal models I and II. The initial values are taken from [25] 